\title{
Estado nutricional de pré-escolares de creche pública: um estudo longitudinal
}

\author{
Nutritional status of childhood of a \\ public day care center: a longitudinal study
}

\author{
Alessandra da Silva Pereira'1, Natasha Gabrielle de Araújo Peixoto², \\ José Firmino Nogueira Neto ${ }^{3}$, Haydée Serrão Lanzillotti ${ }^{4}$, Eliane de Abreu Soares ${ }^{5}$
}

\begin{abstract}
Resumo
O objetivo do estudo foi monitorar o estado nutricional de pré-escolares de uma creche pública. Trata-se de um estudo longitudinal, antes $\left(\mathrm{t}_{0}\right)$ e depois $\left(\mathrm{t}_{1}\right)$, com 51 crianças de 2 a 4 anos de idade. Os procedimentos foram: avaliação antropométrica (P/I, P/E e E/I - OMS 2006, classificação SISVAN-2008); avaliação bioquímica (colesterol total, HDLc, LDLc, triglicerídeos e glicose); avaliação hematológica (hemoglobina); avaliação dietética (pesagem direta dos alimentos e registro alimentar). Para determinação de energia, carboidratos, lipídeos, proteína, fibra alimentar, cálcio, ferro, vitaminas A e C, utilizou-se software Diet Pro, 4.0. Utilizando-se o Índice de Adequação (IA= média do consumo/ EAR ou AI), avaliou-se a adequação do consumo alimentar. As ações em nutrição foram: modificações nos cardápios, atividades pedagógicas, oficina culinária e palestras com os pais e equipe da creche. Observou-se redução de prevalência para déficit E/I e P/I, redução da prevalência de anemia, aumento do consumo de energia, carboidratos, proteínas, vitaminas A e C, ferro e redução discreta do cálcio. Energia, ferro, cálcio e vitamina C apresentaram probabilidade de adequação $\geq 50 \%$. Concluiu-se que se torna importante o monitoramento do estado nutricional de crianças pré-escolares atendidos em creches públicas, bem como ações de educação nutricional.
\end{abstract}

Palavras-chave: pré-escolar; estado nutricional; transtornos da nutrição infantil.

\begin{abstract}
The aim of the study was to monitor the nutritional status of preschool of public day care center. It is a longitudinal study, before $\left(t_{0}\right)$ and after $\left(t_{1}\right)$, with 51 children from 2 to 4 years old. The procedure were: anthropometric evaluation (W/A, W/H and H/A - OMS 2006 - SISVANs, 2008 Classification), blood exams (Total Cholesterol, HDLc, LDLc, triglycerides and glucose), hematological evaluation (hemoglobin) and dietetic evaluation (direct weighing food and dietary register). To calculate energy, carbohydrates, fat, fiber, calcium, iron, A and C vitamins, was used software Diet Pro, 4.0. An adequate Index was calculated ( $\mathrm{Al}=$ consumer medium/ EAR ou $\mathrm{Al}$ ) to evaluate de adequacy of food consumer. The nutrition acts were: day care center menus modification, pedagogic activities, culinary offices, and discussion with parents and school team. We could observe a reduction in prevalence of nutritional deficit $\mathrm{H} / \mathrm{A}$ and W/A, reduction on prevalence of anemia, increases on consumer of: energy, carbohydrates, protein, $\mathrm{A}$ and $\mathrm{C}$ vitamins, iron and decrease on consumer of calcium were observed. Energy, iron, calcium and $\mathrm{C}$ vitamin showed a probability of adequacy $\geq 50 \%$. Inadequacy of fats consume was observed in both moments. We conclude that monitor the status nutritional of preschool children attending in public day care centers is important as well the education nutrition actions.
\end{abstract}

Keywords: preschool; nutritional status; child nutricional disorders.

\footnotetext{
Trabalho realizado na Secretaria Municipal de Educação da Prefeitura de Paraty - Paraty (RJ), Brasil.

${ }^{1}$ Especialista em Nutrição Clínica pela Universidade Gama Filho - Rio de Janeiro (RJ), Brasil.

${ }^{2}$ Nutricionista da Secretaria Municipal de São João da Barra (RJ), Brasil.

${ }^{3}$ Doutor em Fisiopatologia Clínica e Experimental pela Universidade do Estado do Rio de Janeiro (UERJ) - Rlo de Janeiro (RJ), Brasil.

${ }^{4}$ Doutorado em Saúde Coletiva pela UERJ; Professor-Associado da UERJ - Rio de Janeiro (RJ), Brasil.

${ }^{5}$ Doutorado em Ciências dos Alimentos pela Universidade de São Paulo (USP), São Paulo (SP); Professora-Associada da UERJ - Rio de Janeiro (RJ), Brasil

Endereço para correspondência: Alessandra da Silva Pereira - Departamento de Nutrição Fundamental da Escola de Nutrição - Avenida Pasteur, 296 -

CEP: 22290-240 - Rio de Janeiro (RJ), Brasil - E-mail: aspnutri@gmail.com

Fonte de financiamento: Fundação de Amparo à Pesquisa do Estado do Rio de Janeiro (FAPERJ).

Conflito de interesse: nada a declarar.
} 


\section{INTRODUÇÃO}

A fase pré-escolar, em que se tem crianças na faixa etária de dois a seis anos de idade, caracteriza-se por um período no qual ocorrem diversas modificações do padrão alimentar de uma criança. Hábitos alimentares errôneos nessa faixa etária podem causar problemas nutricionais em curto e em longo prazo, tais como: anemia, déficit ou excesso ponderal, hipertensão arterial, diabetes, câncer, entre outras ${ }^{1-4}$.

Além dos fatores nutricionais, outros podem influenciar no crescimento e desenvolvimento das crianças, destacando-se as condições socioeconômicas e demográficas das famílias, os aspectos psicossociais e a história de saúde da criança e da mãe $e^{5,6}$.

Mediante o estilo de vida contemporâneo da população, com aumento da inserção da mulher no mercado de trabalho e consequente modificações no padrão alimentar ${ }^{7}$, as creches estão se tornando uma alternativa assistencial de crianças em idade pré-escolar, tornando-se, muitas vezes, responsáveis pela maior parte da alimentação oferecida diariamente. Nesse cenário, o monitoramento do estado nutricional de crianças, bem como a implantação de ações de Educação Nutricional (EN) são ferramentas necessárias à Vigilância Nutricional. O diagnóstico, a intervenção e o monitoramento do estado nutricional por meio de um conjunto de indicadores socioeconômicos, antropométricos, bioquímicos, hematológicos e de consumo alimentar podem ser instrumentos eficazes e necessários para possíveis intervenções precoces de distúrbios nutricionais ${ }^{7.8}$.

Poucos são os estudos longitudinais que associam frequência à creche e estado nutricional de crianças". O objetivo deste trabalho foi monitorar o estado nutricional de pré-escolares frequentadores de uma creche pública em Paraty, cidade localizada ao sul do Estado do Rio de Janeiro.

\section{MÉTODOS}

Estudo longitudinal, com crianças de dois a quatro anos, frequentadoras de creche pública de Paraty, de março a novembro de 2008.

O projeto foi aprovado pelo COEP/UERJ, sob o número 004.3.2008 e somente participaram pré-escolares cujos responsáveis assinaram o termo de consentimento livre e esclarecido.

Realizaram-se duas avaliações do estado nutricional dos pré-escolares: momento diagnóstico $\left(\mathrm{T}_{0}\right)$ e seis meses após $\left(\mathrm{T}_{1}\right)$, contemplando-se a avaliação antropométrica, bioquímica, hematológica e dietética. Por meio de trabalhos consolidados na literatura, constatou-se que, em seis meses, é possível observar significativas mudanças nos perfis antropométricos, bioquímicos e dietéticos ${ }^{10,11}$.

Para a avaliação antropométrica, utilizou-se a técnica recomendada pela $\mathrm{OMS}^{12}$ : mensuração do peso pela manhã com crianças vestindo o mínimo de roupas e descalças, utilizando balança digital Tanita modelo UM 080W. A Estatura foi mensurada utilizando Estadiômetro Alturexata ${ }^{\circledR}$, com intervalo de $1 \mathrm{~mm}$, estando as crianças, em pé, descalças, com os braços estendidos ao longo do corpo, com a cabeça sem adornos e mantida em plano Frankfurt ${ }^{12}$. Os índices utilizados para o diagnóstico foram: Peso/Idade (P/I), Peso/Altura (P/A) e Altura/Idade (A/I); com referência da OMS (12) e adotada pelo Ministério da Saúde ${ }^{13}$. Para a classificação do estado nutricional, utilizaram-se pontos de corte do SISVAN/ MS $2008^{13}$. Para análise dos resultados antropométricos, os dados foram dicotomizados em: déficit estatural (E/I): <escore- $Z$ - 3 muito baixa estatura para a idade e $\geq$ escore- $Z-3$ e $<$ escore $Z-2$ - baixa estatura para a idade; déficit ponderal (P/I e P/E): <escore Z -3 magreza acentuada e $\geq$ escore Z - 3 e <escore $Z-2$ - magreza e excesso ponderal: >escore $Z+1$ $\mathrm{e} \leq$ escore $Z+2$ risco de sobrepeso, > escore $Z+2$ e $\leq$ escore $Z+3$ sobrepeso, > escore Z +3 - obesidade.

Avaliação bioquímica: colesterol total e frações, triglicerídeos e glicose. Para realização das análises, utilizou-se o Analisador Automático A15 (BioSystems ${ }^{\circledR}$ ) e kits de reativos específicos (Biosystems ${ }^{\circledR}$ ). O LDL-colesterol (low density cholesterol) foi calculado conforme cálculo de Friedewald. As amostras de sangue foram coletadas em tubo apropriado, contendo ativador de coágulo. No dia anterior a coleta de sangue, as mães foram orientadas quanto ao jejum prévio de 12 horas de seus filhos.

A Classificação de dislipidemias foi fundamentada na I Diretriz de Prevenção da Aterosclerose na infância e adolescência da Sociedade Brasileira de Cardiologia (SBC) ${ }^{11}$ e glicemia de jejum utilizou pontos de corte adotados pela Sociedade Brasileira de Diabetes $(\mathrm{SBD})^{14}$. Crianças que apresentaram alterações nos seus perfis bioquímicos receberam orientações nutricionais específicas.

Por meio da avaliação hematológica, pesquisou-se a anemia ferropriva, considerada quando valor de hemoglobina inferior a $11 \mathrm{~g} / \mathrm{dL}^{15}$. As crianças com anemia foram encaminhadas ao posto de saúde para suplementação de sulfato ferroso, bem como receberam orientações nutricionais para anemia, como parte da intervenção nutricional realizada.

No inquérito dietético, utilizou-se Pesagem Direta dos Alimentos (PDA) consumidos na creche, durante três dias não consecutivos, complementado por Registro Alimentar (RA) e aplicado com os responsáveis da criança $a^{16}$. A pesagem dos alimentos foi realizada usando balança Plenna ${ }^{\circledR}$ modelo Apollo, com capacidade máxima de $5 \mathrm{~kg}$ e graduação de $1 \mathrm{~g}$.

Alimentos sólidos e preparações que compunham as refeições foram servidos em pratos individuais, devidamente identificados e previamente pesados. Os líquidos foram medidos 
em Becker. No caso de repetição da refeição, forneceu-se o porcionamento estabelecido. No caso de resto, aplicou-se o percentual de participação de cada preparação/alimento. No caso de escolha seletiva, considerou-se o alimento em questão consumido integralmente, sendo calculado o percentual de participação dos outros alimentos na mescla de alimentos da refeição. Todos os dados foram registrados em um formulário. O consumo alimentar individual de cada preparação foi obtido com o uso da fórmula:

Consumo alimentar individual da preparação = porção oferecida - resto

Uma entrevista com os responsáveis permitiu realizar o registro dos alimentos e bebidas ingeridos no dia da pesquisa, antes da chegada da criança à creche. Os dados foram coletados pela pesquisadora ou por bolsista previamente treinada $\mathrm{e}$ registrados em formulário próprio. Analogamente, os alimentos consumidos em domicílio, após a permanência na creche, foram anotados pelos pais. Todos os alimentos e preparações alimentares foram registrados em medidas caseiras e convertidos em gramas, de acordo com Soares, Portella e Ishii ${ }^{17}$. Em caso de dúvidas, os pais eram consultados.

Avaliação quantitativa da composição nutricional da dieta (energia, carboidratos, lipídeos totais, proteínas, fibras alimentares, cálcio, ferro, vitaminas A e C) foi realizada com auxílio do Programa Diet Pro versão 5.0 ${ }^{18}$.

Para avaliação da dieta, criou-se um Índice de Adequação (IA), em que o numerador foi representado pela média do consumo de energia ou nutriente e o denominador pela Estimated Average Requirement (EAR) ou Adequate Intake $(\mathrm{AI})^{19-22}$. Nutrientes com índice abaixo de 1: consumo inadequado. Esses representam consumo abaixo de P50 na curva de referência do nutriente, ou seja, apresentam 0,50 de probabilidade de adequação ou menos. Os índices acima de 1 até o Upper Level (UL) foi considerado como consumo adequado. Os valores encontrados foram comparados às DRI's para a faixa etária de 1 a 4 anos incompletos.

No momento diagnóstico, coletaram-se informações sociodemográficas das famílias das crianças, utilizando-se questionário já validado ${ }^{23} \mathrm{e}$ adaptado, acrescentando dados de histórico de saúde.

Após o diagnóstico, implementaram-se ações em nutrição, estimulando hábitos alimentares saudáveis, como alterações nos cardápios da instituição, com maior oferta de frutas, legumes e verduras, leguminosas e cereais. Ações de EN para uma alimentação saudável foram desenvolvidas regularmente com as merendeiras da creche, com o objetivo de estimular práticas de preparo saudáveis com menos utilização de gorduras e açúcares.
Com a equipe pedagógica, realizaram-se quatro encontros de 30 minutos. No primeiro, relataram-se os objetivos do projeto. No segundo, divulgou-se o resultado da avaliação do estado nutricional das crianças. No terceiro, ressaltaram-se as peculiaridades da alimentação infantil e a importância da equipe para a formação de hábitos alimentares saudáveis. No último encontro, a pesquisadora ministrou uma palestra sobre "Alimentação saudável". Os recursos pedagógicos utilizados para fixação dos conteúdos foram apostila e folders.

Os responsáveis pelas crianças participaram de três encontros bimestrais, com duração média de 20 minutos cada. $\mathrm{Na}$ primeira reunião, foram expostos os resultados da pesquisa, destacando-se os distúrbios nutricionais observados. No segundo encontro, objetivou-se sensibilizar os responsáveis em relação aos aspectos mais importantes da alimentação infantil. No terceiro encontro, apresentaram-se os aspectos de uma alimentação saudável e se destacou, também, alguns alimentos típicos da região, que são possíveis de serem introduzidos na dieta de seus filhos.

As atividades de EN com as crianças foram quinzenais, com duração de 15 a 20 minutos, realizadas nos horários de recreação. Foram desenvolvidas atividades, tais como "reconhecendo" e "conhecendo" os alimentos. No primeiro ("reconhecendo"), o objetivo foi conhecer e listar os alimentos com os quais as crianças possuíam familiaridade; no segundo ("conhecendo"), o objetivo foi apresentar novos alimentos para que as crianças descobrissem seus aspectos sensoriais, como cor, textura e sabor. As crianças realizaram duas oficinas de preparação de receitas. Complementando as ações de nutrição, as crianças visitaram um hortifrutigranjeiro da região e assistiram à apresentação de um teatro de fantoches, cujos personagens eram os alimentos.

Após seis meses das ações em nutrição desenvolvidas na creche $\left(\mathrm{T}_{1}\right)$, realizaram-se os mesmos procedimentos utilizados na fase diagnóstica $\left(\mathrm{T}_{0}\right)$.

O tratamento estatístico foi realizado por meio de análise descritiva dos dados incluindo medidas de posição, de dispersão e frequências de ocorrências. $O$ teste de Kolmogorov-Smirnov foi usado para verificar a normalidade da distribuição das matrizes das variáveis dietéticas medidas em escala intervalar. Para o comportamento não gaussiano da distribuição amostral, utilizou-se de teste não paramétrico de Wilcoxon para avaliar grandeza das diferenças entre as matrizes dos dados de consumo alimentar; teste exato de Fisher para estimar probabilidade de ocorrência das frequências com número reduzido de observações e teste de aderência do $\chi^{2}$ para verificar discrepâncias entre as frequências observadas e esperadas. 


\section{RESULTADOS}

Das 51 crianças que fizeram parte do estudo desde o seu início, 46 (90,20\%) participaram das duas avaliações antropométricas, havendo perda de amostra de 9,8\%. Na avaliação bioquímica e na hematológica, a perda de amostra atingiu $25 \%$, participando das duas fases 38 crianças. A maior perda de amostra $(29,4 \%)$ foi constatada no inquérito dietético, com participação de 36 crianças ao final do estudo. As perdas se devem principalmente ao alto nível de migração característico do município. O perfil sociodemográfico e de saúde estão descritos na Tabela 1.
Os resultados da avaliação antropométrica, antes e após intervenção, encontram-se sumarizados na Tabela 2.

Na Tabela 2, há a indicação de redução de prevalência para déficit estatural (E/I) e ponderal (P/I) significativos. Embora não significativo, houve aumento discreto $(4,3 \%)$ da prevalência de excesso ponderal (P/I), após a intervenção.

A avaliação bioquímica possibilitou calcular a prevalência de dislipidemias entre as crianças no momento diagnóstico e após a intervenção (Tabela 3).

Por meio do teste exato de Fisher, revelou-se não haver diferença significativa entre as prevalências de colesterol

Tabela 1. Características sociodemográficas e de história de saúde de pré-escolares assistidos na creche pública de Paraty, RJ, 2008

\begin{tabular}{|c|c|c|c|c|c|}
\hline Variável & n & $\%$ & Variável & $\mathbf{n}$ & $\%$ \\
\hline Tipo de construção da residência Alvenaria & & & Escolaridade de quem conhece bem a criança & & \\
\hline Abastecimento de água & 51 & 100 & Até 8 anos & 28 & 54,9 \\
\hline Rede Pública & 50 & 98 & 9 a 11 anos & 20 & 39,2 \\
\hline Outras & 1 & 2 & $>11$ anos & 3 & 5,9 \\
\hline Coleta de esgoto & & & Situação matrimonial & & \\
\hline Rede Pública & 20 & 39 & Vive com o companheiro & 40 & 78,4 \\
\hline Fossa Adequada & 30 & 59 & Não vive com o companheiro & 11 & 21,6 \\
\hline Outros & 1 & 2 & Renda per capita & & \\
\hline Coleta de lixo regular & 51 & 100 & até 1 salário mínimo & 43 & 84,3 \\
\hline Número de membros da família & & & de 1 a 2 salários mínimos & 4 & 7,8 \\
\hline 2 a 4 & 33 & 64,7 & Mais de 2 salários mínimos & 2 & 3,9 \\
\hline$\geq 5$ & 18 & 35,3 & Não soube informar & 2 & 3,9 \\
\hline Números de cômodos por residência & & & Prematuridade & & \\
\hline$<2$ & 6 & 11,8 & Sim & 7 & 17,7 \\
\hline 2 a 4 & 37 & 72,6 & Não & 44 & 82,3 \\
\hline$\geq 5$ & 8 & 15,7 & Peso ao nascer (gramas) & & \\
\hline Número de pessoas por dormitório & & & $<1.500$ & - & - \\
\hline 2 a 4 & 45 & 88,2 & $<2.500$ & 18 & 35,3 \\
\hline$\geq 5$ & 6 & 11,8 & $2.500-3.999$ & 27 & 52,9 \\
\hline Números de cômodos por residência & & & $\geq 4.000$ & 3 & 5,9 \\
\hline$<2$ & 6 & 11,8 & $\mathrm{Nsi}^{*}$ & 3 & 5,9 \\
\hline 2 a 4 & 37 & 72,6 & Amamentação exclusiva & & \\
\hline$\geq 5$ & 8 & 15,7 & não amamentou & 3 & 5,9 \\
\hline Número de pessoas por dormitório & & & $<1$ mês & 7 & 13,7 \\
\hline 2 a 4 & 45 & 88,2 & -3 meses & 5 & 9,8 \\
\hline$\geq 5$ & 6 & 11,8 & 3-5 meses & 22 & 43,1 \\
\hline Chefe da família & & & $\geq 5$ meses & 10 & 19,6 \\
\hline Pai & 32 & 627 & $\mathrm{Nsi}^{*}$ & 4 & 7,8 \\
\hline Mãe & 11 & 216 & Cartão vacinação atualizado & & \\
\hline Outros & 0 & 1,0 & Sim & 50 & 98 \\
\hline $\begin{array}{l}\text { Uutros } \\
\text { Nível de escolaridade defe da família }\end{array}$ & 0 & 15,1 & Não & 1 & 2 \\
\hline Nivel de escolaridade do chefe da família & & & Parasitose intestinal & & \\
\hline até 8 anos & 30 & 58,8 & Sim & 14 & 27,4 \\
\hline 9 a 11 anos & 18 & 35,3 & Não & 36 & 72,6 \\
\hline$>11$ anos & 3 & 5,9 & Assistência médica regular & & \\
\hline Membro da família que conhece bem os hábitos da & & & Sim & 23 & 45,2 \\
\hline criança & & & Não & 28 & 54,8 \\
\hline Pai & 2 & 3,9 & Doenças crônicas & & \\
\hline Mãe & 42 & 82,4 & Sim & 18 & 35,3 \\
\hline Outros & 7 & 13,7 & Não & 33 & 64,7 \\
\hline
\end{tabular}

*Nsi: Não soube informar 
Tabela 2. Comparação entre as prevalências observadas antes e após ações em nutrição, de acordo com a classificação SISVAN (2008), para os indicadores E/I, P/E, P/I e IMC

\begin{tabular}{|c|c|c|c|}
\hline \multirow[b]{2}{*}{ Classificação } & \multicolumn{2}{|c|}{ Prevalência } & \multirow[b]{2}{*}{$\mathrm{p}-\chi^{2}$} \\
\hline & $\begin{array}{c}\text { Diagnóstico } \\
\left(\mathrm{T}_{0}\right) \\
\mathrm{n}(\%)\end{array}$ & $\begin{array}{c}\text { Após intervenção } \\
\left(\mathrm{T}_{1}\right) \\
\mathrm{n}(\%)\end{array}$ & \\
\hline Estatura/Idade ${ }^{*}$ & & & 0,0007 \\
\hline Déficit estatural & $30(65,2)$ & $13(28,3)$ & \\
\hline Adequado & $16(34,8)$ & $33(71,7)$ & \\
\hline Peso/Idade ${ }^{*}$ & & & 0,007 \\
\hline Déficit ponderal & $12(26,1)$ & $0(0)$ & \\
\hline Adequado & $34(73,9)$ & $45(97,8)$ & \\
\hline Excesso ponderal & $0(0)$ & $1(2,2)$ & \\
\hline Peso/Estatura & & & 0,5807 \\
\hline Déficit ponderal & $0(0)$ & $0(0)$ & \\
\hline Adequado & $35(76,1)$ & $34(73,9)$ & \\
\hline Excesso ponderal & $11(23,9)$ & $12(26,1)$ & \\
\hline
\end{tabular}

${ }^{*}$ Estatisticamente significativo; $\mathrm{n}=46$ total $(\mathrm{p}=0,7283), \mathrm{LDL}$ colesterol $(\mathrm{p}=0,3712)$ e triglicerídeos $(\mathrm{p}=0,1534)$, antes e após seis meses. Nenhuma criança apresentou glicemia elevada em ambos os momentos do estudo. No entanto, quando estudadas as diferenças de concentrações séricas pelos valores medianos, antes e depois da intervenção, para colesterol total $\left(\mathrm{T}_{0}=139 \mathrm{mg}\right.$ e $\left.\mathrm{T}_{1}=148 \mathrm{mg} ; \mathrm{p}=0,0715\right), \mathrm{LDL}$ colesterol $\left(\mathrm{T}_{0}=80 \mathrm{mg}\right.$ e $\left.\mathrm{T}_{1}=90 \mathrm{mg} ; \mathrm{p}=0,0152\right)$ e triglicerídeos $\left(\mathrm{T}_{0}=66 \mathrm{mg}\right.$ e $\left.\mathrm{T}_{1}=66 \mathrm{mg} ; \mathrm{p}=0,6377\right)$, observou-se diferença significativa apenas para LDL colesterol.

$\mathrm{Na}$ avaliação hematológica, o teste de Wilcoxon demonstrou diferença significativa entre as medianas $(11,9 \mathrm{~g} / \mathrm{dL}$ e $12,2 \mathrm{~g} / \mathrm{dL}$, respectivamente, $\mathrm{p}=0,0004)$. As prevalências de anemia em $t_{0}$ e $t_{1}$ foram: 10,5 e 5,3\%, respectivamente.

Com os inquéritos dietéticos, revela-se o perfil de consumo alimentar e adequação da dieta das crianças frequentadoras da creche (Tabela 4). A fim de analisar diferenças significativas em $\mathrm{T}_{0}$ e $\mathrm{T}_{1}$, aplicou-se, nas matrizes de consumo das variáveis dietéticas, o teste de Kolmogorov-Smirnov $(p<0,10)$ para verificar se as distribuições tinham a feição gaussiana.

Tabela 3. Comparação das prevalências de dislipidemias antes e após ações em nutrição

\begin{tabular}{lcccc} 
& \multicolumn{2}{c}{$\begin{array}{c}\text { Diagnóstico } \\
\left(\mathrm{T}_{0}\right)\end{array}$} & \multicolumn{2}{c}{$\begin{array}{c}\text { Após a intervenção } \\
\left(\mathrm{T}_{1}\right)\end{array}$} \\
\cline { 2 - 5 } Indicadores $^{*}$ & $\begin{array}{c}\text { Sujeitos } \\
\mathbf{n}(\%)\end{array}$ & $\begin{array}{c}\text { Prevalência } \\
\mathbf{n}(\%)\end{array}$ & $\begin{array}{c}\text { Sujeitos } \\
\mathbf{n}(\%)\end{array}$ & $\begin{array}{c}\text { Prevalência } \\
\mathbf{n}(\%)\end{array}$ \\
$\begin{array}{l}\text { Colesterol total } \\
\quad \text { Limítrofe: } 150-169 \mathrm{mg} / \mathrm{dL}\end{array}$ & 6 & 15,38 & 8 & 20,5 \\
$\quad$ Alto: $\geq 170 \mathrm{mg} \mathrm{dL}$ & 10 & 25,64 & 9 & 23,1 \\
\hline $\begin{array}{l}\text { LDL Colesterol } \\
\quad \text { Limítrofe: } 100-129 \mathrm{mg} / \mathrm{dL} \\
\quad \text { Alto: } \geq 130 \mathrm{mg} / \mathrm{dL}\end{array}$ & 4 & 10,26 & 4 & 28,2 \\
\hline $\begin{array}{l}\text { Triglicerídeos } \\
\quad \text { Limítrofe: } 100-129 \mathrm{mg} / \mathrm{dL} \\
\quad \text { Alto: } \geq 130 \mathrm{mg} / \mathrm{dL}\end{array}$ & 4 & 10,26 & 7 & 10,3 \\
\hline
\end{tabular}

*Sociedade Brasileira de Cardiologia ${ }^{11} ; n=39$

Tabela 4. Adequação da dieta ingerida pelos pré-escolares antes e após ações em nutrição

\begin{tabular}{lcccccccc} 
& \multicolumn{2}{c}{ Consumo médio } & \multicolumn{3}{c}{ Consumo mediano } & \multicolumn{3}{c}{ Índice de Adequação } \\
\cline { 2 - 8 } Energia/nutriente & $\mathrm{T}_{0}$ & $\mathrm{~T}_{1}$ & $\mathrm{~T}_{0}$ & $\mathrm{~T}_{1}$ & Valor $\mathbf{p}$ & $\mathrm{T}_{0}$ & $\mathrm{~T}_{1}$ & $\mathrm{Valor} \mathbf{p}$ \\
\cline { 2 - 8 } Energia & $1223 \pm 136$ & $1447 \pm 146$ & 1156 & 1465 & $0,0064^{*}$ & 1,09 & 1,15 & $0,2304^{*}$ \\
Carboidratos (g) & $195,61 \pm 26,53$ & $231,56 \pm 31,53$ & 184,63 & 228,64 & $0,008^{*}$ & 1,7 & 2,11 & $0,0144^{*}$ \\
Proteína (g) & $33,05 \pm 6,37$ & $46,27 \pm 5,75$ & 42,90 & 54,61 & $0,0033^{*}$ & 3,96 & 5,04 & $0,0057^{*}$ \\
Ferro (mg) & $5,93 \pm 0,85$ & $6,371 \pm 0,71$ & 5,61 & 6,36 & 0,1161 & 0,96 & 1,09 & 0,1732 \\
Cálcio (mg) & $630,68 \pm 134,53$ & $559,42 \pm 114,95$ & 577,11 & 506,13 & 0,1126 & 1,15 & 1,01 & 0,067 \\
Vitamina C (mg) & $28,57 \pm 8,77$ & $76 \pm 9,04$ & 33,66 & 24,59 & $0,0387^{*}$ & 2,69 & 1,97 & $0,0146^{*}$ \\
Vitamina A (meq/ret) & $403,83 \pm 86,69$ & $419,05 \pm 480,42$ & 368,61 & 362,61 & 0,6591 & 1,47 & 1,45 & 0,8536 \\
Fibra (g) & $11,06 \pm 1,36$ & $12,11 \pm 1,53$ & 11,17 & 12,26 & 0,1233 & 0,59 & 0,65 & 0,1704 \\
\hline
\end{tabular}

*Diferença significativa; $\mathrm{n}=37$ 
Por meio do teste, descartou-se a hipótese de normalidade para ferro e vitamina $\mathrm{C}$ e se afirmou tal comportamento para as distribuições de energia, carboidratos, proteina, vitamina A e fibra alimentar. Esses achados conduziram à utilização do teste de Wilcoxon para verificar a significância das diferenças encontradas. Analogamente, replicou-se o procedimento para as matrizes dos índices de adequação das variáveis dietéticas, sendo indicado o comportamento gaussiano para carboidratos, proteínas e vitamina A. Energia, ferro, cálcio e vitamina C não mostraram tal comportamento, conduzindo-se também ao teste de Wilcoxon. Esse cuidado prende-se à alta variabilidade que apresentam os dados dietéticos, exigindo-se maior rigor na análise estatística.

Com relação aos lipídeos totais da dieta, o percentual em relação ao valor energético total (VET) foi de $23,95 \% \pm 1,13$ e $20,33 \pm 1,19 \%$ antes e após intervenção. Em ambos os momentos, $100 \%$ das crianças apresentaram inadequação, pois o percentual deveria estar entre 30 e $40 \%$. O percentual de proteínas no $\mathrm{T}_{0}$ e $\mathrm{T}_{1}$, em relação ao VET, foi de 10,8 e de 12,8\%, respectivamente, enquanto que os carboidratos apresentaram distribuição de 65,2 e de $66,9 \%$.

\section{DISCUSSÃO}

O déficit estatura (E/I) 1 foi expressivamente alto (65,2\%) em $\mathrm{T}_{0}$ (Tabela 2), superando os dados obtidos pela PNDS, $2006^{24}$, para essa faixa etária na região Sudeste $(13,4 \%)$. Barbosa, Salles Costa e Soares ${ }^{25}$ em estudo longitudinal com 35 pré-escolares de creche filantrópica de Paquetá, RJ, observaram pequena redução nas prevalências de risco nutricional (2,9\%), entre os dois momentos do estudo, diferindo do presente trabalho que demonstrou redução de $36,9 \%$ nas prevalências de déficit estatural. $O$ déficit ponderal, segundo $\mathrm{P} / \mathrm{I}$, apresentou prevalência de 26,1\%. Barbosa, Salles Costa e Soares ${ }^{25}$ observaram prevalência de $25,7 \%$ de crianças em risco nutricional, havendo redução de $100 \%$ após seis meses de monitoramento, assemelhando-se ao presente estudo.

Com relação ao $\mathrm{P} / \mathrm{E}$, a prevalência de excesso ponderal em $\mathrm{T}_{0}$ e $\mathrm{T}_{1}$ foi de $23,9 \%$ e de $26,1 \%$, respectivamente. Não foi observado caso de déficit ponderal segundo esse indicador. Os resultados do presente estudo assemelham-se aos encontrados por Barbosa, Salles Costa e Soares ${ }^{25}$, que observaram prevalências similares na fase diagnóstica (25,7\%). Porém, houve maior redução na prevalência no segundo momento $(17,2 \%)$ após seis meses de monitoramento.

Em relação aos exames bioquímicos, são poucos os estudos que abordam as prevalências de dislipidemias em pré-escolares, dificultando essa discussão. Silva et al. ${ }^{26} \mathrm{em}$ pesquisa realizada com crianças de dois a nove anos de idade, frequentadores do
Hospital Universitário Antonio Pedro (HUAP) da Universidade Federal Fluminense, em Niterói, observaram prevalência de dislipidemias, considerando-se valores limítrofes e alterados, de $41,6 \%$ (CT) , 32,9\% (LDLc) e 23,1\% (triglicerídeos), assemelhando-se aos presentes achados. Grillo et al. ${ }^{27}$, contando com a participação de 257 escolares de 3 a 14 anos de idade, sadios, frequentadores de centros institucionalizados na cidade de Itajaí, SC, observaram prevalências de 23,7, 18,8 e 4,7\% para CT, LDLc e triglicerídeos, respectivamente, considerando valores limítrofes e altos.

Observou-se redução nas prevalências para concentrações altas com consequente aumento nas concentrações limítrofes, tanto para triglicerídeos, quanto para CT, indicando transição entre dislipidemia e normalidade (Tabela 3). Houve diferença significativa nos valores medianos da concentração sérica de triglicerídeos, que aumentaram após seis meses $(\mathrm{p}=0,0152)$. Esse achado é de extrema relevância, uma vez que mostra a premência de se confrontar as refeições realizadas na creche e as realizadas em domicílio, dando-se ênfase aos fins de semana, quando os pais são mais liberais em relação à alimentação de seus filhos. Barbosa, Soares e Lanzillotti ${ }^{28}$, quando compararam o consumo alimentar durante a semana com o fim de semana, concluíram que a dieta do fim de semana é mais inadequada do que a dieta oferecida às crianças durante a semana, já que se reduz o consumo de leguminosas, frutas e legumes.

A prevalência de anemia no início do estudo $\left(\mathrm{T}_{0}\right)$ foi de $10,5 \%$, menor quando comparada a outros estudos. Almeida et al. ${ }^{29}$ estudaram 184 crianças de creches de Jardinópolis, SP, e constataram 29,3\% de anemia nelas. Em estudo de Matta et al. ${ }^{30}$ a prevalência alcançou $47,3 \%$ em 617 crianças de 29 creches públicas do Rio de Janeiro. No presente estudo, a prevalência de anemia após seis meses, diminuiu de 10,5\% para 5,3\%, ou seja, redução em torno de $50 \%$. Após o momento diagnóstico do estudo $\left(\mathrm{T}_{0}\right)$, além das ações de Educação Nutricional, os responsáveis foram orientados a levar as crianças ao posto de saúde da região, para suplementação ferrosa.

$\mathrm{O}$ aumento no consumo mediano de macronutrientes e consequentemente de energia pode estar relacionado à evolução da faixa etária das crianças uma vez que, no inicio do estudo, os pré-escolares encontravam-se na faixa de dois anos enquanto que, no segundo, próximos a faixa de três anos e seis meses. Tuma, Costa e Schmitz ${ }^{31}$, em pesquisa com 230 pré-escolares de creche da cidade de Brasília, observaram consumo médio de $1310 \pm 135 \mathrm{kcal}$ de energia, não sendo muito diferente do encontrado no presente estudo (Tabela 4). Barbosa et al..$^{32} \mathrm{em}$ estudo longitudinal realizado com crianças na faixa etária de 2 a 3 anos, em creche filantrópica da cidade de Paquetá, observaram aumento no consumo médio de energia e proteína entre os dois momentos do estudo, assemelhando-se aos presentes achados. 
O aumento discreto no consumo de fibras alimentares (Tabela 4) pode estar relacionado ao maior consumo de hortaliças, frutas e leguminosas ofertadas nos cardápios da creche. Barbosa et al..$^{32}$, corroborando com o presente estudo, observaram melhora significativa no consumo de fibras, após intervenção nutricional com pré-escolares de creche em Paquetá.

O consumo mediano de ferro aumentou, apesar de não significativo, podendo estar relacionado à maior oferta de alimentos ricos em ferro heme nos cardápios da creche. Cabe ressaltar que o índice de adequação do consumo de ferro, que estava abaixo do esperado em $\mathrm{T}_{0}$, ultrapassou o desejado em $\mathrm{T}_{1}$. Barbosa et al. ${ }^{32}$ também constataram maior consumo de ferro em seu estudo. A melhoria do perfil de prevalência de anemia no grupo em apreço pode estar também relacionada ao aumento significativo do consumo de vitamina $\mathrm{C}$ durante o almoço, devido à introdução de frutas e/ou suco de frutas, embora a adequação desse nutriente tivesse sido observada anteriormente. Ghoneim, Hassan e Amine ${ }^{33}$, em estudo longitudinal realizado com 935 crianças de três creches, na cidade de Alexandria (Egito), verificaram que quase $40 \%$ das crianças consumiam ferro e vitamina $\mathrm{C}$ acima ou igual a recomendação da ingestão.

O consumo mediano de cálcio diminuiu após seis meses, podendo estar relacionado ao menor consumo de leite uma vez que, no início do estudo, muitas crianças tomavam leite de vaca em diversos horários do dia. Mas é importante notar que os índices de adequação são os desejáveis.

Os IA de vitamina A ultrapassaram $50 \%$ em ambos os momentos. Um leve aumento no consumo mediano, após intervenção nutricional, pode estar relacionado à introdução de alimentos fontes nos cardápios da creche, tais como fígado bovino, mamão, cenoura, entre outros.

O estudo limita-se pela falta de grupo controle, uma vez que o município só apresentava uma única creche. Além disso, dados escassos sobre o perfil antropométrico da população caiçara limitou a discussão sobre a avaliação antropométrica das crianças.

\section{CONCLUSÃO}

Após seis meses de monitoramento do estado nutricional dos pré-escolares, verificou-se melhoria nos perfis antropométrico, hematológico e dietético. O perfil de lipídeos sanguíneos é o que mais denota preocupação para as ações intervencionistas dietéticas, necessitando-se dilatar o tempo para o emprego de estratégias de educação nutricional e monitoramento de resultados. Essa conjunção de ações possibilitará atingir, de forma holística, os indicadores estabelecidos nos protocolos nacionais que objetivam a segurança nutricional para pré-escolares.

Conclui-se que as ações em nutrição realizadas foram particularmente eficientes em alcançar níveis de adequação das variáveis dietéticas.

\section{AGRADECIMENTOS}

À Fundação Carlos Chaga Filho de Amparo à Pesquisa do Estado do Rio de Janeiro (FAPERJ) e ao Laboratório de Lipídeos (LABLIP) por todo o apoio.

\section{REFERÊNCIAS}

1. Bissoli MC, Lanzillotti HS. Educação Nutricional como forma de intervenção: avaliação de uma proposta para pré-escolares. Rev Nutr. 1997;2(10):107-13.

2. Pellanda LC, Echenique L, Barcellos LMA, Maccari J, Borges FK, Zen BL. Doença cardíaca isquêmica: a prevenção inicia durante a infância. J Pediatr. 2002;78(2):91-6.

3. Pinheiro ARO, Freitas SFT, Corso ACT. Uma abordagem epidemiológica da obesidade. Rev Nutr. 2004;17(4):523-33.

4. Maddah M, Mohtasham-Amiri Z, Rashid A, Karandish M. Height and weight of urban preschool children in relation to their mother's educational levels and employment status in Rasht City, northern Iran. Matern Child Nutr. 2007;3(1):52-7.

5. Abidoye RO, Sikabofori L. A study of prevalence of protein energy malnutrition among 0-5 years in rural Benue State, Nigéria. Nutr Health. 2000;13(4):235-47.

6. Escoda MSQ. Para a crítica da transição nutricional. Ciênc saúde Coletiva. 2002;7(2):219-26. Disponível em http://www.scielo.br/pdf/csc/ v7n2/10241.pdf
7. Instituto Brasileiro de Geografia e Estatística (IBGE). Pesquisa de Orçamentos Familiares, 2002-2003. Aquisição domiciliar per capita, Brasil e grandes regiões. Rio de Janeiro; 2004.

8. Medronho RA, Bloch KV, Luiz RR, Werneck GL. Epidemiologia. São Paulo: Atheneu; 2007.

9. Pereira AS, Lanzillotti HS, Soares EA. Frequência à creche e estado nutricional de pré-escolares: uma revisão sistemática. Rev paul pediatr. 2010;28(4):366-72.

10. Taddei JA, Cannon MJ, Warner L, Souza P, Vitalle S, Palma D, et al. Nutritional gains of underprivileged children attending a day care center in S. Paulo City, Brazil: a nine month follow-up study. Rev Bras Epidemiol. 2000;3(1-3):29-37.

11. Brasil. Sociedade Brasileira de Cardiologia. I Diretriz de Prevenção da Aterosclerose na Infância e Adolescência. Arq Bras Cardiol. 2005;85(6):1-36.

12. World Health Organization (WHO). Child Growth Standards: Methods and development: lenght/height - for-age, weight-for-age, weight-forlenght, weight-for-height and body mass index-for-age. Geneva: WHO; 2006. Disponível em: http://www.who.int/childgrowth/standaeds/en/ 
13. Brasil. Ministério da Saúde. Sistema de Vigilância Alimentar e Nutricional (SISVAN): Orientações para a coleta, o processamento, a análise de dados e a informação em serviços de saúde. Brasília: Ministério da Saúde; 2008.

14. Brasil. Sociedade Brasileira de Diabetes. Tratamento e acompanhamento do diabetes mellitus. Diretrizes da Sociedade Brasileira de Diabetes (SBD). São Paulo: SBD; 2007.

15. World Health Organization (WHO). The clinical use of blood in Medicine, Obstetrics \& Anaesthesia, Trauma \& Burns. Geneva: WHO; 2001 .

16. Cavalcanti AAM, Priore SE, Franceschini SCC. Estudos de consumo alimentar: aspectos metodológicos gerais e o seu emprego na avaliação de crianças e adolescentes. Rev Bras Saúde Matern Infant. 2004;3(4):229-40.

17. Soares EA, Portella ES, Ishii M. Relação de medidas caseira de 320 alimentos e respectivas gramaturas. São Paulo, Ceane; 1991.

18. Bressan, J. Sistemas de suporte à Avaliação Nutricional e Prescrições de dietas. Agromídia Software - DietPro, 5ª ed., CD-ROM; 2008.

19. Food and Nutrition Board/Institute of Medicine. Dietary reference intake for energy, carbohydrate, fat, fatty acids, cholesterol, protein and amino acids. Washington D.C.: National Academies Press; 2005.

20. Food and Nutrition Board/Institute of Medicine. Dietary reference intake for calcium, phosphorus, magnesium, vitamin D and fluoride. Washington D.C.: National Academies Press; 1997.

21. Food and Nutrition Board/Institute of Medicine. Dietary reference intake for vitamin $\mathrm{C}$, vitamin $\mathrm{E}$, selenium and carotenoids. Washington D.C.: National Academies Press; 2005.

22. Food and Nutrition Board/Institute of Medicine. Dietary reference intake for vitamin $\mathrm{A}$, vitamin $\mathrm{K}$, arsenic, boron, chromium, copper, iodine, iron, manganese, molybdenium, nickel, silicon, vanadium and zinc. Washington D.C.: National Academies Press; 2002. 773 p.

23. Saldiva SRDM. Enteroparasitoses, indicadores antropométricos e inquérito alimentar em crianças de uma localidade rural do Estado de São Paulo [dissertação mestrado]. São Paulo: Departamento de Epidemiologia da Faculdade de Saúde Pública da Universidade de São Paulo; 1995.
24. Centro brasileiro de análise e planejamento. Pesquisa Nacional de Demografia e Saúde da Criança e da Mulher. Brasil, 2008. Disponível em: http://www.cebrap.org.br

25. Barbosa RMS, Salles Costa R., Soares EA. Estado nutricional de crianças atendidas em uma creche filantrópica: estudo longitudinal de 6 meses. Rev Alim Nutr. 2007;18(2):215-21.

26. Silva RA, Kanaan S, Silva LE, Peralta RHS. Estudo do perfil lipídico em crianças e jovens do ambulatório pediátrico do hospital universitário Antônio Pedro associado ao risco de dislipidemias. J Bras Patol Med Lab. 2007;43(2):95-101.

27. Grillo LP, Crispim SP, Siebert NA, Andrade ATW, Rossi A, Campos IS. Perfil lipídico e obesidade em escolares de baixa renda. Rev Bras Epidemiol. 2005;8(1):78-81.

28. Barbosa RMS, Soares EA, Lanzillotti HS. Avaliação da ingestão de nutrientes de crianças de uma creche filantrópica: aplicação do consumo dietético de referência. Rev bras saúde matern infant. 2007;7(2):159-66

29. Almeida CAN, Ricco, RG, Ciampo LA, Souza AM, Pinho AP, Oliveira JED. Fatores associados a anemia por deficiência de ferro em crianças pré-escolares brasileiras. J Pediatr. 2004;80(3):229-34.

30. Matta IEA, Veiga GL, Baião MR, Santos MMAS, Luiz RR. Anemia em crianças menores de cinco anos que freqüentam creches públicas do município do Rio de Janeiro, Brasil. Rev Bras Saúde Matern Infant. 2005;5(3):349-57.

31. Tuma RCFB, Costa THM, Schmitz BAS. Avaliação antropométrica e dietética em três creches de Brasília, Distrito Federal. Rev Bras Saúde Matern Infant. 2005;5(4):419-28.

32. Barbosa RMS, Croccia C, Carvalho CGN, Franco VC, Salles-Costa R, Soares EA, Consumo alimentar de crianças com base na pirâmide alimentar brasileira infantil. Rev Nutr. 2007;18(5):633-41.

33. Ghoneim EH, Hassan MH, Amine EK. An intervention programme for improving the nutritional status of children aged 2-5 years in Alexandria. J East Mediterr Health. 2004;10(6):828-43.

Recebido em: 10/05/2013 Aprovado em: 17/06/2013 